\title{
Effects of $17 \beta$-estradiol and raloxifene on endothelial OPG and RANKL secretion
}

\author{
Wojciech Karwowski ${ }^{1}$, Katarzyna Lekesiz ${ }^{2}$, Ewa Koc-Żórawska ${ }^{3}$, Krzysztof Wnuczko ${ }^{1}$, \\ Hanna Borysewicz-Sanczyk ${ }^{4}$, Beata Naumnik ${ }^{1}$ \\ ${ }^{1}$ Arciszewscy Polyclinic — Gynecology and Obstetrics, District Hospital, Bialystok, Poland

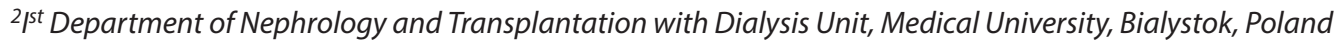 \\ ${ }^{3} / I^{\text {nd }}$ Department of Nephrology and Hypertension with Dialysis Unit, Medical University, Bialystok, Poland \\ ${ }^{4}$ Department of Pediatrics, Endocrinology, Diabetology with Cardiology Divisions, University Children's Clinical Hospital of Bialystok
}

\begin{abstract}
Objectives: This study aims to asses the effects of estradiol vs. raloxifene on the levels of osteoprotegerin and soluble Receptor Activator of Nuclear Factor kB Ligand (sRANKL) in Human Umbilical Vein Endothelial Cells (HUVEC) culture in standard and calcifying medium.

Material and methods: Human Umbilical Vein Endothelial Cells were isolated from human umbilical vein by standard method. The supernatant concentrations of osteoprotegerin (OPG) and sRANKL (ELISA) were determined after incubation with glicerophosphate, estradiol , raloxifene, glicerophoshate and estradiol, glicerophosphate and raloxifene in comparison with control group at four designated time points (0, 1, 2 and 4 days of incubation).

Results: Incubation of estradiol with HUVEC colony lowered the OPG level significantly after day 2 and 4 . Meantime, the level of sRANKL was stable. Raloxifene added to standard growth medium also significantly lowered OPG concentration after day 4 only, with no impact on sRANKL concentration. When added to calcifying medium, both estradiol and raloxifene significantly changed OPG level during the experiment. In all treated groups OPG levels were lower than in groups exposed to calcifying medium only. Neither estradiol, nor raloxifene changed sRANKL levels during the experiment.
\end{abstract}

Conclusions: Estradiol and raloxifene affect OPG secretion from endothelial cells in vitro which may suggest their modifying role in pathogenesis of vascular calcification in postmenopausal women.

Key words: vascular calcification, OPG, sRANKL, menopause, estrogens, raloxifene

Ginekologia Polska 2017; 88, 4: 167-173

\section{INTRODUCTION}

It has been proved that during the calcification of vessels occur similar processes to those in bone tissue biomineralization. In depositions located in both lamina interna and media of the vessel wall, matrix vesicles were identified [1]. Samples from the walls of calcified vessels as well as in vitro studies revealed the expression of macromolecular matrix proteins involved in the regulation of biomineralization, i.e.: bone morphogenetic protein -2 (BMP-2), osteopontin, matrix gla protein (MGP), osteonectin, collagen, osteocalcin , and the osteoprotegerin/receptor activator factor NF-KB/ receptor activator factor NF-KB ligand system (OPG/RANK/RANKL) [2-4].
Over the last few years intensive studies have been carried out to assess the OPG/RANK/RANKL system contribution to vascular calcification (VC) process. Osteoprotegerin appears to represent the molecular link between bone resorption and vascular calcification, and may help to understand the high prevalence of atherosclerosis and osteoporosis in postmenopausal women. Serum OPG is potentially an independent predictor of early vascular adverse changes in osteoporotic postmenopausal women [5]. Helas showed that unopposed RANKL activity in osteoprotegerin-deficient mice resulted in osteoporosis and vascular calcification. RANKL inhibition by denosumab reduced 
vascular calcification in prednisolon-induced osteoporosis in mice, which is further evidence for the link between the bone and vascular system [6].

Estrogen therapy for postmenopausal women reduces vascular calcification [7-9]. It has been shown that postmenopausal women with a higher serum estradiol (E2) level had a reduced coronary artery calcium score independent of age and other coronary risk factors. Possibly, estradiol lowers the calcified-plaque burden of coronary arteries in postmenopausal women [10]. This is due to the indirect effect on calcification risk factors as well as direct genomic and extragenomic effect on macrophages, endothelial cells and vascular smooth muscle cells (VSMCs). Estradiol has been found to modulate the secretion of matrix proteins (osteopontin, BSP, MGP, RANKL/OPG), attenuates proliferation and differentiation of VSMCs as well as the activity of calcifying cells $[7,11,12]$.

Raloxifene is a non-steroid, selective estrogen receptor modulator (SERM) demonstrating, depending on target tissue, either agonistic or antagonistic effects. Raloxifene therapy involves beneficial impact on cardiovascular risk factors: increases concentration of HDL-cholesterol, and decreases level of LDL-cholesterol, fibrynogen, homocysteine, and lower intensity of inflammatory processes [7, 13-17]. Raloxifene, used in postmenopausal osteoporosis therapy, affects the synthesis and release of intercellular matrix proteins, proliferation and differentiation of VSMCs [12] and has a protective effect on vascular endothelium by, among others, increased expression of eNOS [18-19].

Animals subjected to ovariectomy demonstrated substantial increase of calcium deposit accumulation in the vessel wall as well as an increase of the OPG/RANKL ratio, primarily resulting from a decrease of RANKL concentration (as opposed to the bone system) [20]. Clinical trials have established that raloxifene therapy significantly lowers the level of RANKL and temporally decreases the level of OPG (which normal level is restored after a year of therapy). Main store of OPG is the bones and cardiovascular system. It is still unclear whether the influence of raloxifene on the concentration of OPG and RANKL in serum is mainly due to its osteoprotective effect, or to the processes occurring in the vessel wall [21].

The increase of osteoporosis parallel to the development of calcification in postmenopausal women proves that the role of female sex hormones is vital for the regulation of deposition and resorption processes in bone tissue. Despite long-term research, the influence of hormone replacement therapy on the cardiovascular system is still subject to debate. The mechanisms involving the reducing effect of SERM (i.e. raloxifene) on cardiovascular events rate in postmenopausal need to be deeply examined.

\section{The aim of the study}

Direct effects of raloxifene on endothelial OPG/ /RANK/RANKL system have not yet been studied. Therefore, we decided to test that $17 \beta$-estradiol and raloxifene would influence endothelial in vitro secretion of OPG/RANKL in calcifying milieu which may support this way for modifying vascular calcification.

\section{MATERIAL AND METHODS}

\section{Chemicals}

$17 \beta$-estradiol, raloxifene hydrochloride, $\beta$-glycerophosphate disodium salt hydrate, dimethyl sulfoxide (DMSO) were purchased from Sigma Chemical (St. Louis, MO).

\section{Cell culture - Human Umbilical Vein Endothelial Cells (HUVEC)}

Human umbilical vein obtained from healthy adult woman was cannulated and filled with $0.1 \%$ collagenase solution (Sigma-Aldrich, USA), then incubated for $10 \mathrm{mi}$ nutes at $37^{\circ} \mathrm{C}$. After incubation, the vein was perfused with HBSS solution without $\mathrm{Ca}++$ and $\mathrm{Mg}++$ (Sigma-Aldrich, USA). Cells were collected from the perfusate by centrifugation $\left(200 \mathrm{~g}, 4^{\circ} \mathrm{C}\right)$, and suspended in Medium 199 containing $15 \%$ bovine calf serum, $100 \mathrm{U} / \mathrm{mL}$ penicillin $\mathrm{G}, 100 \mu \mathrm{g} / \mathrm{mL}$ streptomycin, $0.25 \mu \mathrm{g} / \mathrm{mL}$ amphotericine $\mathrm{B}, 100 \mu \mathrm{g} / \mathrm{mL}$ low molecular weight heparin and $200 \mu \mathrm{g} / \mathrm{mL}$ Endothelial Cell Growth Supplement, buffered with 25mM HEPES (all reagents Sigma-Aldrich, USA). Cells were cultured in fibronectin precoated flasks (Nalge Nunc International, USA). Primary culture was passaged after washing and trypsinization. Cells from third subcultures were used for the experiments.

All cell cultures were maintained in a humidified $5 \%$ $\mathrm{CO}_{2}$ atmosphere at $37^{\circ} \mathrm{C}$. Human Umbilical Vein Endothelial Cells (HUVEC) were divided into groups and incubated with glicerophosphate (group 2), raloxifene (3), estradiol (4), glicerophosphate and estradiol (5), glicerophosphate and raloxifene (6), and control group (1), which received solvent $\left(10^{-9} \mathrm{~m} / \mathrm{L}\right.$ DMSO) used for estradiol and raloxifene. That day, when we administered substances described above was called day 0 . Concentrations of raloxifene and estradiol were established on the basis of literature for efficacy at physiological levels [22]. Medium was changed at day 1,2 and 4. Supernatants from examined and control HUVEC were collected on days 1, 2, and 4 for determination of protein concentrations. All samples were frozen at $-80^{\circ} \mathrm{C}$ till the day of measurements.

\section{Laboratory procedures}

Human OPG and soluble RANKL (sRANKL) levels were measured in HUVEC supernatants using sandwich-type enzyme-linked immunosorbent assay (ELISA) kits according 
to the manufacturers' instructions. The human OPG ELISA kit was purchased from RayBio, and human SRANKL kits were purchased from Tecomedical Group. Note of, the ELISA assay for human sRANKL from Tecomedical Group detects only uncomplexed soluble RANKL.

\section{Statistical analysis}

The median, 1 and 3 quartiles values were calculated for each group of data. The results were evaluated by using the Mann-Whitney rank-sum test and post-hoc Conover test. Correlation were calculated by the Spearman's method. Statistical significance was defined as $p$ less than 0.05 . All analyses were carried out by using Statistica software (version 10.0 PL, StatSoft, Tulsa, OK, USA).

\section{RESULTS}

\section{Control group}

Concentration of OPG and SRANKL was determined at each of the four designated time points (Tab. 1, Tab. 2). There was no significant difference in concentration of OPG at each of the designated time points but there was a tendency to decrease the OPG level during the experiment (Fig. 1). The level of sRANKL increased statistically at day 4 comparing with day 0 and $1\left(p_{0 v s 4}=0.001, p_{1 v s 4}=0.003\right.$ ) (Fig. 2). There was no significant correlation between OPG and SRANKL concentration (day $1: R=-0.5, p=0.24$, day $2: R=-0.09$, $p=0.84$, day $4: R=-0.07, p=0.81)$.

\section{The effect of glicerophosphate on OPG and SRANKL secretion}

OPG levels showed a significant and time-dependent linear decrease in supernatants from HUVEC exposure to medium containing excess phosphate $\left(\mathrm{p}_{0 \mathrm{vs} 4}=0.03\right.$ and $\left.p_{1 v s 4}=0.01\right)$. Comparing to the control group OPG concentration was statistically lower in glicerophosphate group on day 1,2 and 4 ( $p=0.037, p=0.004$ and $p=0.001$ respectively) (Fig. 1). There were no significant difference in sRANKL concentration in cultures containing glicerophosphate (Fig. 2).

Table 1. Concentration of OPG (pg/mL) in the supernatants of control HUVEC and in cells incubated with analyzed substances

\begin{tabular}{|l|c|c|c|c|}
\hline OPG $(\mathbf{p g} / \mathbf{m L})$ & Day 0 & Day 1 & Day 2 & Day 4 \\
\hline Control group & 32.88 & 36.42 & 31.13 & 28.09 \\
\hline Glicerophosphate & $\left(\mathrm{Q}_{1}-30.2, \mathrm{Q}_{3}-7.01\right)$ & $\left(\mathrm{Q}_{1}-33.3, \mathrm{Q}_{3}-39.4\right)$ & $\left(\mathrm{Q}_{1}-27.5, \mathrm{Q}_{3}-35.4\right)$ & $\left(\mathrm{Q}_{1}-23.9, \mathrm{Q}_{3}-32.7\right)$ \\
\hline Estradiol & 25.76 & 30.74 & 25.7 & 20.96 \\
\hline & $\left(\mathrm{Q}_{1}-25.6, \mathrm{Q}_{3}-34.6\right)$ & $\left(\mathrm{Q}_{1}-21.5, \mathrm{Q}_{3}-32.2\right)$ & $\left(\mathrm{Q}_{1}-21.2, \mathrm{Q}_{3}-27.4\right)$ & $\left(\mathrm{Q}_{1}-15.6, \mathrm{Q}_{3}-23.9\right)$ \\
\hline Raloxifene & 27.85 & 29.85 & 26.02 & 23,12 \\
\hline Glicero+ Estradiol & $\left(\mathrm{Q}_{1}-27.1, \mathrm{Q}_{3}-33.2\right)$ & $\left(\mathrm{Q}_{1}-26.6, \mathrm{Q}_{3}-35.61\right)$ & $\left(\mathrm{Q}_{1}-25.4, \mathrm{Q}_{3}-27.4\right)$ & $\left(\mathrm{Q}_{1}-16.2, \mathrm{Q}_{3}-25.5\right)$ \\
\hline Glicero+Raloxifene & 30.01 & 30.04 & 28.07 & 18.74 \\
\hline
\end{tabular}

Abbreviations: Glicero — glicerophosphate

Table 2. Concentration of sRANKL (pmol/L) in the supernatants of control HUVEC and in cells incubated with analyzed substances

\begin{tabular}{|c|c|c|c|c|}
\hline sRANKL (pmol/L) & Day 0 & Day 1 & Day 2 & Day 4 \\
\hline Control group & $\begin{array}{c}0.12 \\
\left(Q_{1}-0.04, Q_{3}-0.16\right)\end{array}$ & $\begin{array}{c}0.14 \\
\left(Q_{1}-0.04, Q_{3}-0.18\right)\end{array}$ & $\begin{array}{c}0.17 \\
\left(Q_{1}-0.09, Q_{3}-0.19\right)\end{array}$ & $\begin{array}{c}0.22 \\
\left(Q_{1}-0.17, Q_{3}-0.26\right)\end{array}$ \\
\hline Glicerophosphate & $\begin{array}{c}0.15 \\
\left(Q_{1}-0.10, Q_{3}-0.32\right)\end{array}$ & $\begin{array}{c}0.23 \\
\left(Q_{1}-0.06, Q_{3}-0.68\right)\end{array}$ & $\begin{array}{c}0.18 \\
\left(Q_{1}-0.09, Q_{3}-0.22\right)\end{array}$ & $\begin{array}{c}0.22 \\
\left(Q_{1}-0.18, Q_{3}-0.44\right)\end{array}$ \\
\hline Estradiol & $\begin{array}{c}0.17 \\
\left(Q_{1}-0.11, Q_{3}-0.49\right)\end{array}$ & $\begin{array}{c}0.09 \\
\left(Q_{1}-0.07, Q_{3}-0.25\right)\end{array}$ & $\begin{array}{c}0.18 \\
\left(Q_{1}-0.11, Q_{3}-0.19\right)\end{array}$ & $\begin{array}{c}0.2 \\
\left(Q_{1}-0.16, Q_{3}-0.24\right)\end{array}$ \\
\hline Raloxifene & $\begin{array}{c}0.11 \\
\left(Q_{1}-0.07, Q_{3}-0.29\right)\end{array}$ & $\begin{array}{c}0.11 \\
\left(Q_{1}-0.07, Q_{3}-0.27\right)\end{array}$ & $\begin{array}{c}0.14 \\
\left(Q_{1}-0.09, Q_{3}-0.17\right)\end{array}$ & $\begin{array}{c}0.19 \\
\left(Q_{1}-0.14, Q_{3}-0.30\right)\end{array}$ \\
\hline Glicero+ Estradiol & $\begin{array}{c}0.18 \\
\left(Q_{1}-0.12, Q_{3}-0.23\right)\end{array}$ & $\begin{array}{c}0.27 \\
\left(Q_{1}-0.10, Q_{3}-0.57\right)\end{array}$ & $\begin{array}{c}0.18 \\
\left(Q_{1}-0.14, Q_{3}-0.19\right)\end{array}$ & $\begin{array}{c}0.19 \\
\left(Q_{1}-0.17, Q_{3}-0.22\right)\end{array}$ \\
\hline Glicero+Raloxifene & $\begin{array}{c}0.11 \\
\left(Q_{1}-0.09, Q_{3}-0.24\right)\end{array}$ & $\begin{array}{c}0.2 \\
\left(Q_{1}-0.06, Q_{3}-0.39\right)\end{array}$ & $\begin{array}{c}0.13 \\
\left(Q_{1}-0.09, Q_{3}-0.17\right)\end{array}$ & $\begin{array}{c}0.2 \\
\left(Q_{1}-0.17, Q_{3}-0.22\right)\end{array}$ \\
\hline
\end{tabular}

Abbreviations: Glicero — glicerophosphate 


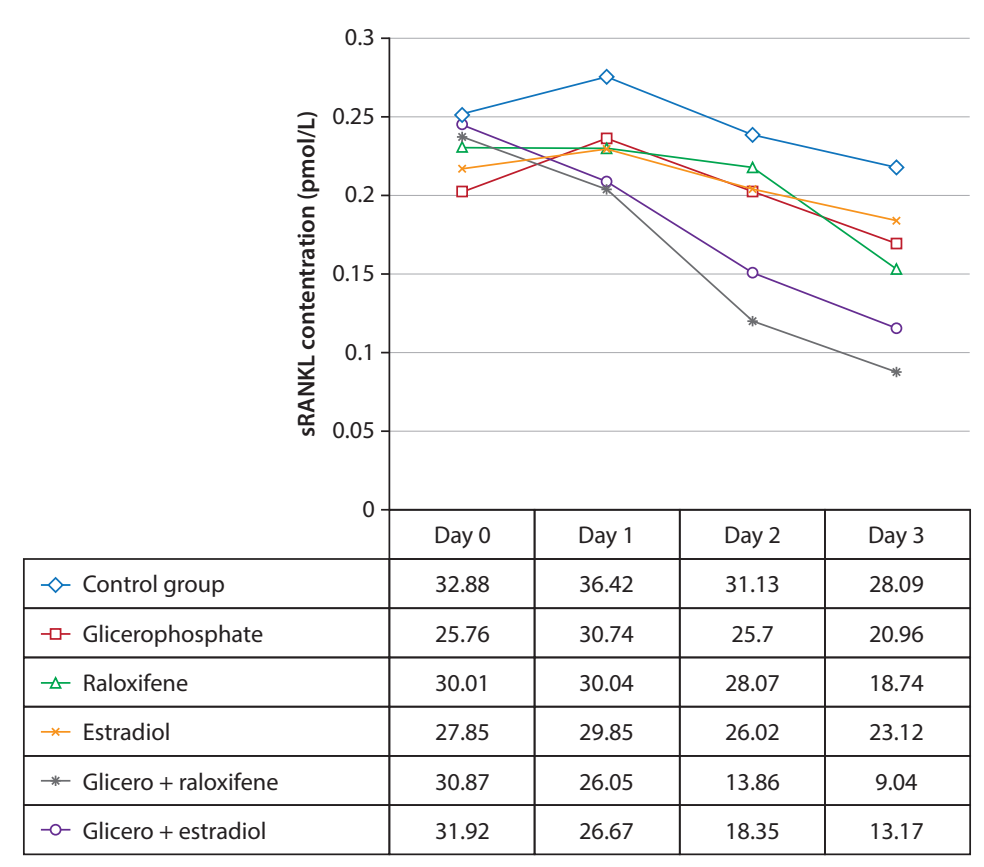

Figure 1. Concentration of osteoprotegerin (OPG) in the supernatants of HUVEC

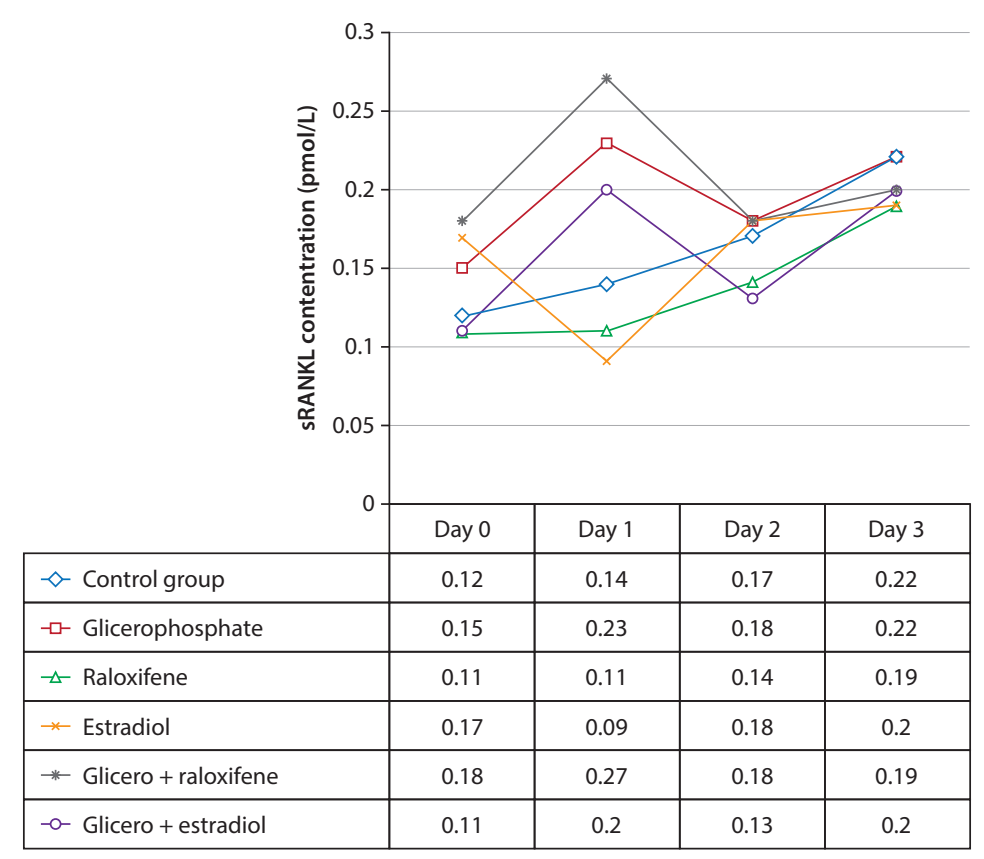

Figure 2. Concentration of SRANKL in the supernatants of HUVECs

\section{The effect of estradiol and raloxifene treatment on resting endothelial OPG and SRANKL secretion}

Incubation of estradiol with HUVEC colony lowered the OPG concentration significantly on day 4 when compared with day 0 and 1 ( $p=0.0009$ and $p=0.0004$, respectively). Comparing to control group OPG secretion was significantly lower on day $4(p=0,006)$ (Fig. 1). The level of sRANKL after a fall at day 1 , showed a tendency to increase on day 2 and 4 . Comparing to control group estradiol did not affect sRANKL levels (Fig. 2).

Raloxifene added to standard growth medium significantly lowered OPG level on day 4 only ( $p=0,008$ ) (Fig. 1). sRANKL levels decreased on day 1 , and then showed a tendency to increase in groups exposed to medium containing raloxifene. Comparing to the control group raloxifene 
significantly lowered OPG concentration on day $4(p=0.0009)$ with no impact on sRANKL level (Fig. 1 and 2).

\section{The effect of estradiol and raloxifene treatment on OPG and SRANKL expression in HUVEC exposed to calcifying medium}

When added to calcifying medium, both estradiol and raloxifene significantly changed OPG level during the experiment. In all treated groups OPG concentration were lower than in groups exposed to calcifying medium only, reaching a statistically significant difference on day 2 and 4 ( $p<0.05$ ) (Fig. 1). When comparing with groups containing glicerophosphate, raloxifene significantly lowered the OPG level on day 4 only. Meantime, estradiol lowered OPG concentration on day 2 and 4 .

Neither estradiol nor raloxifene changed sRANKL levels during the experiment (Fig. 2).

\section{DISCUSSION}

Evidence from the last years have drawn attention to the relationship between vascular calcification (VC) and bone health. The mechanisms involved in VC are still not fully understood but recent studies have shown that RANK/RANKL/OPG plays an important role in regulating those processes. The exact functions of these molecules in relation to VC are still unclear. OPG is produced by VSMCs and may be upregulated by inflammatory modulators or downregulated by anti-inflammatory factors [23]. OPG is also produced by ECs and may serve as an EC survival signal to oppose the pro-apoptotic actions of TRAIL.

Elevated OPG levels may exert an anti-calcific effect within the vasculature, protect against arterial damage and VC, or reflect a general state of EC dysfunction. However, the protective role of OPG found in the animal model has not been observed in humans. In contrast, in humans many authors suggest a positive association between serum OPG and atherosclerosis, arterial calcification, or CV disease [24]. High serum concentrations of OPG were correlated to the severity of peripheral arterial disease [25] and heart failure [26], unstable angina [27], acute myocardial infarction and risk of death [28]. Bjerre [29] reports that increased levels of OPG are not directly involved with the extent of myocardial injury. The author also reports that high levels of OPG would be a biomarker for cardiovascular complications.

RANKL may be considered as an important factor in the pathogenesis of VC, but a definitive relationship between RANKL and bone-vascular axis in CKD patients remains unclear. Only few studies reported that RANKL is related to cardiovascular events. RANKL secreted by ECs is able to activate RANK receptor and promote an osteogenic differentiation of VSMCs [7]. Cardiovascular events and mortality are significantly higher in these patients with low serum
sRANKL levels. Lieb et al. [30] reported that serum RANKL concentrations is not related to carotid arteries calcification (CAC), the incidents of CVD or mortality. Higher levels of total RANKL were inversely correlated with CAC and triglycerides in postmenopausal woman [31]. Samelson concluded, that RANKL inhibition with denosumab treatment had no effect on progression of aortic calcification or incidence of adverse cardiovascular events in postmenopausal women with osteoporosis and high cardiovascular risk [32].

There is a significant association between BMD loss and vascular calcifications in the aorta in postmenopausal women. Low estradiol levels could be involved in the development of both coronary calcification and BMD loss. There are several pathophysiological mechanisms that could explain the role that estrogens play in vascular calcification inhibition. Firstly, estradiol prevents atherosclerotic plaque development [33]. Administration of E2 decreases VSMC proliferation in animal and human models, through activation of nitric oxide synthase and through decreased mitogen-induced VSMC proliferation. Secondly, VSMC and endothelial cells express RANK, RANKL and OPG, and therefore can respond to RANKL stimulation. RANKL induces VC through an increase in bone morphogenetic protein and a decrease in matrix Gla protein, an inhibitor of VC. Estradiol is able to attenuate RANKL-induced VC [7, 33]. Therefore, through differential actions in the expression of key proteins, E2 preserves the original contractile VSMCs features, decreasing transdifferentiation towards a calcifying phenotype.

To the best of our knowledge this is the first study presenting the influence of calcification-promoting culture conditions and female sex hormones on the OPG and SRANKL release in vitro. Under standard conditions, stable levels of osteoprotegerin and gradually increasing concentration of soluble RANKL were observed throughout the experiment. Substantial changes have also been shown in the release of the proteins under unfavorable, calcification-promoting culture conditions, as well as in cultures subjected to hormone stimulation. Under hyperphosphatemic conditions, OPG concentrations dropped steadily with each day of the experiment, while sRANKL values remained stable. The addition of estradiol or raloxifene had no effect on OPG and sRANKL concentrations.

In the control group osteoprotegerin concentration was stable while the values of sRANKL exhibited significant linear increase. Changes in concentrations of the analyzed substances may be related to physical shortcomings of the culture. Lekesiz, using the same HUVEC culture model (but observed only over several hours) hasfound the increase in OPG release and the drop in SRANKL secretion by endothelial cells [34]. In our study, differences in OPG release may result from the time of measurement: most probably, 
certain unfavorable physical and mechanical factors trigger the secretion of Weibel-Palade bodies content, which in turn causes temporary rise in OPG concentration followed by its drop before the protein is produced de novo.

It is still not completely clear whether concentration of OPG in the blood can serve as a marker of the intensity of the atherosclerotic process, a mediator of its progression, or an indicator of natural defense against calcification of the vascular wall. In our experiment calcification-promoting conditions caused lower OPG concentrations with stable levels of sRANKL. Since we did not use an hourly observation protocol, it is difficult to predict the early changes in the concentration of analyzed substances. Decreasing OPG concentrations may be explained either by excessive OPG release in the early hours of the experiment and its further exhaustion (which would be in line with Lekesiz's findings), or by the neutralization of OPG by means of its binding to sRANKL.

The in vitro studies have shown that estradiol or raloxifene maintain VSMCs' OPG production when cultured under elevated levels of calcium phosphates [22]. We have confirmed that under calcification-promoting conditions, endothelial cells change the SRANKL and OPG release. In normophosphatemic conditions, both estradiol and raloxifene caused a decrease in concentrations of OPG, which suggested either reduced production or increased consumption of that protein.

Unlike VSMCs, both drugs used in the experiment did not prevent a hyperphosphatemia-induced drop in OPG concentration. In normal, as well as when cultured under elevated levels of calcium phosphates conditions, we found lower OPG concentration in EC colony treated with E2 or raloxifene This may suggest that endothelial cells respond differently than VSMCs especially under unfavorable living conditions. However, in order to deeply determine the profile of OPG/sRANKL secretion by endothelial cells we would need to determine this response in different period of time that is shorter and longer than the culture originally designed by our team. Another thing that would also be worth considering is the assessment of other factors that seem to be important for the initiation of the vascular calcification process as well as mRNA expression of those proteins.

\section{CONCLUSIONS}

In conclusion, we found that estriadiol and raloxifene decreased OPG secretion and did not maintain endothelial cell OPG production when cultured under elevated levels of calcium phosphates. Serum OPG concentration, seen in many cardiovascular pathology, seems to be dependent on VSMCs rather than EC release.

Despite osteoprotegerin is being a potent inhibitor of bone loss, higher serum levels of this cytokine are marker of calcification-related vascular diseases. A deeper knowledge of the mechanisms involved in the association between OPG serum levels, bone integrity and cardiovascular disease can provide important data for future therapeutic interventions.

\section{REFERENCES}

1. Tanimura A, McGregor DH, Anderson HC. Matrix vesicles in atherosclerotic calcification. Proc Soc Exp Biol Med. 1983; 172(2): 173-177, indexed in Pubmed: 6828462.

2. Byon $\mathrm{CH}$, Chen Y. Molecular Mechanisms of Vascular Calcification in Chronic Kidney Disease: The Link between Bone and the Vasculature. Curr Osteoporos Rep. 2015; 13(4): 206-215, doi: 10.1007/s11914-0150270-3, indexed in Pubmed: 25947259.

3. Liu W, Zhang Y, Yu CM, et al. Current understanding of coronary artery calcification. J Geriatr Cardiol. 2015; 12(6): 668-675, doi: 10.11909/j. issn.1671-5411.2015.06.012, indexed in Pubmed: 26788045

4. Wu PC, Zheng CM, Liao MT, et al. Bone turnover and vascular calcification. J Nephrol Ther. 2014; 4: 171, doi: 10.4172/2161-0959.1000171.

5. Shargorodsky $M$, Boaz $M$, Luckish $A$, et al. Osteoprotegerin as an independent marker of subclinical atherosclerosis in osteoporotic postmenopausal women. Atherosclerosis. 2009; 204(2): 608-611, doi: 10.1016/j. atherosclerosis.2008.10.024, indexed in Pubmed: 19062016.

6. Helas S, Goettsch C, Schoppet M, et al. Inhibition of receptor activator of NF-kappaB ligand by denosumab attenuates vascular calcium deposition in mice. Am J Pathol. 2009; 175(2): 473-478, doi: 10.2353/ajpath.2009.080957, indexed in Pubmed: 19590040.

7. Karwowski W, Naumnik B, Szczepański M, et al. The mechanism of vascular calcification - a systematic review. Med Sci Monit. 2012; 18(1): RA1-R11, doi: 10.12659/msm.882181, indexed in Pubmed: 22207127.

8. Mackey RH, Kuller LH, Sutton-Tyrrell K, et al. Hormone therapy, lipoprotein subclasses, and coronary calcification: the Healthy Women Study. Arch Intern Med. 2005; 165(5): 510-515, doi: 10.1001/archinte.165.5.510, indexed in Pubmed: 15767525.

9. Manson JE, Allison MA, Carr JJ, et al. Women's Health Initiative and Women's Health Initiative-Coronary Artery Calcium Study Investigators, WHI and WHI-CACS Investigators. Estrogen therapy and coronary-artery calcification. N Engl J Med. 2007; 356(25): 2591-2602, doi: 10.1056/NEJMoa071513, indexed in Pubmed: 17582069.

10. Jeon GH, Kim SH, Yun SC, et al. Association between serum estradiol level and coronary artery calcification in postmenopausal women. Menopause. 2010; 17(5): 902-907, doi: 10.1097/gme.0b013e3181d76768, indexed in Pubmed: 20512078.

11. Doherty TM, Asotra K, Fitzpatrick LA, et al. Calcification in atherosclerosis: bone biology and chronic inflammation at the arterial crossroads. Proc Natl Acad Sci U S A. 2003; 100(20): 11201-11206, doi: 10.1073/pnas.1932554100, indexed in Pubmed: 14500910.

12. Takahashi $\mathrm{K}$, Ohmichi $\mathrm{M}$, Yoshida $\mathrm{M}$, et al. Both estrogen and raloxifene cause G1 arrest of vascular smooth muscle cells. J Endocrinol. 2003; 178(2): 319-329, indexed in Pubmed: 12904179.

13. Oktem M, Atar I, Zeyneloglu HB, et al. Raloxifene has favourable effects on metabolic parameters but has no effect on left ventricular function in postmenopausal women. Pharmacol Res. 2008; 57(5): 364-368, doi: 10.1016/j.phrs.2008.03.010, indexed in Pubmed: 18467115.

14. Eilertsen AL, Sandvik L, Steinsvik B, et al. Differential impact of conventional-dose and low-dose postmenopausal hormone therapy, tibolone and raloxifene on C-reactive protein and other inflammatory markers. J Thromb Haemost. 2008; 6(6): 928-934, doi: 10.1111/j.1538-7836.2008.02970.x, indexed in Pubmed: 18394014.

15. Choi BG, Vilahur G, Zafar MU, et al. Selective estrogen receptor modulation influences atherosclerotic plaque composition in a rabbit menopause model. Atherosclerosis. 2008; 201(1): 76-84, doi: 10.1016/j. atherosclerosis.2008.01.017, indexed in Pubmed: 18367192.

16. Nanetti L, Camilletti A, Francucci CM, et al. Role of raloxifene on platelet metabolism and plasma lipids. Eur J Clin Invest. 2008; 38(2): 117-125, doi: 10.1111/j.1365-2362.2007.01905.x, indexed in Pubmed: 18226045.

17. Mosca L, Grady D, Barrett-Connor E, et al. Effect of raloxifene on stroke and venous thromboembolism according to subgroups in postmenopausal women at increased risk of coronary heart disease. Stroke. 2009; 40(1): 147-155, doi: 10.1161/STROKEAHA.108.518621, indexed in Pubmed: 18948611.

18. Chan YC, Leung FP, Wong WT, et al. Therapeutically relevant concentrations of raloxifene dilate pressurized rat resistance arteries 
via calcium-dependent endothelial nitric oxide synthase activation. ArteriosclerThromb Vasc Biol. 2010; 30(5): 992-999, doi: 10.1161/ATVBAHA.110.203935, indexed in Pubmed: 20185791.

19. Leung FP, Yung LM, Leung HS, et al. Therapeutic concentrations of raloxifene augment nitric oxide-dependent coronary artery dilatation in vitro. Br J Pharmacol. 2007; 152(2): 223-229, doi: 10.1038/sj.bjp.0707387, indexed in Pubmed: 17618301.

20. Choi BG, Vilahur G, Cardoso L, et al. Ovariectomy increases vascular calcification via the OPG/RANKL cytokine signalling pathway. Eur J Clin Invest. 2008; 38(4): 211-217, doi: 10.1111/j.1365-2362.2008.01930.x, indexed in Pubmed: 18279396.

21. Fernández-García D, Muñoz-Torres M, Mezquita-Raya $P$, et al. Effects of raloxifene therapy on circulating osteoprotegerin and RANK ligand levels in post-menopausal osteoporosis. J Endocrinol Invest. 2008; 31(5): 416-421, indexed in Pubmed: 18560259.

22. Rzewuska-Lech $E$, Jayachandran M, Fitzpatrick LA, et al. Differential effects of 17 beta-estradiol and raloxifene on VSMC phenotype and expression of osteoblast-associated proteins. Am J Physiol Endocrinol Metab. 2005; 289(1): E105-E112, doi: 10.1152/ajpendo.00366.2004, indexed in Pubmed: 15713688.

23. Higgins $C L$, Isbilir $S$, Basto $P$, et al. Distribution of alkaline phosphatase, osteopontin, RANK ligand and osteoprotegerin in calcified human carotid atheroma. Protein J. 2015; 34(5): 315-328, doi: 10.1007/s10930015-9620-3, indexed in Pubmed: 26307009.

24. Sasso GR, Florencio-Silva R, Simões RS, et al. Elevated serum osteoprotegerin levels in women: friend or foe? Rev Assoc Med Bras (1992). 2015; 61(6): 524-529, doi: 10.1590/1806-9282.61.06.524, indexed in Pubmed: 26841162

25. Ziegler S, Kudlacek S, Luger A, et al. Osteoprotegerin plasma concentrations correlate with severity of peripheral artery disease. Atherosclerosis. 2005; 182(1): 175-180, doi: 10.1016/j.atherosclerosis.2005.01.042, indexed in Pubmed: 16115489.

26. Ueland $T$, Yndestad $A$, Dahl CP, et al. TNF revisited: osteoprotegerin and TNF-related molecules in heart failure. Curr Heart Fail Rep. 2012; 9(2): 92-100, doi: 10.1007/s11897-012-0088-6, indexed in Pubmed: 22453763.
27. Aksu F, Ozçelik F, Kunduracilar $\mathrm{H}$, et al. The relation between the levels of osteoprotegerin and the degree of coronary artery disease in patients with acute coronary syndrome and stable angina pectoris. Kardiol Pol. 2014; 72(1): 34-41, doi: 10.5603/KP.2014.0004, indexed in Pubmed: 24469748.

28. Montagnana M, Lippi G, Danese $E$, et al. The role of osteoprotegerin in cardiovascular disease. Ann Med. 2013; 45(3): 254-264, doi: 10.3109/07853890.2012.727019, indexed in Pubmed: 23110639.

29. Bjerre M. Osteoprotegerin (OPG) as a biomarker for diabetic cardiovascular complications. Springerplus. 2013; 2: 658, doi: 10.1186/2193-18012-658, indexed in Pubmed: 24349960.

30. Lieb W, Gona P, Larson MG, et al. Biomarkers of the osteoprotegerin pathway: clinical correlates, subclinical disease, incident cardiovascular disease, and mortality. Arterioscler Thromb Vasc Biol. 2010; 30(9): 1849-1854, doi: 10.1161/ATVBAHA.109.199661, indexed in Pubmed: 20448212.

31. Poornima IG, Mackey RH, Buhari AM, et al. Relationship between circulating serum osteoprotegerin and total receptor activator of nuclear $\mathrm{\kappa}-\mathrm{B}$ ligand levels, triglycerides, and coronary calcification in postmenopausal women. Menopause. 2014; 21(7): 702-710, doi: 10.1097/GME.0000000000000127, indexed in Pubmed: 24473535.

32. Samelson EJ, Miller PD, Christiansen C, et al. RANKL inhibition with denosumab does not influence 3 -year progression of aortic calcification or incidence of adverse cardiovascular events in postmenopausal women with osteoporosis and high cardiovascular risk. J Bone Miner Res. 2014; 29(2): 450-457, doi: 10.1002/jbmr.2043, indexed in Pubmed: 23873632.

33. Andersen $G \varnothing$, Knudsen EC, Aukrust $P$, et al. Elevated serum osteoprotegerin levels measured early after acute ST-elevation myocardial infarction predict final infarct size. Heart. 2011; 97(6): 460-465, doi: 10.1136/hrt.2010.206714, indexed in Pubmed: 21270073.

34. Lekesiz K, Naumnik B, Borysewicz-Sanczyk H, et al. Effect of unfractionated heparin, enoxaparin and sulodexide on the relations between secretion and expression of OPG, RANKL and vWF in HUVEC. Folia Histochem Cytobiol. 2013; 51(2): 156-163, doi: 10.5603/FHC.2013.0016, indexed in Pubmed: 23907946. 